\title{
Analysis of the mathematical modelling of a static expansion system
}

\section{Carlos Mauricio Villamizar Mora ${ }^{1}$, Jonathan Javier Duarte Franco ${ }^{1}$, Victor José Manrique Moreno ${ }^{1}$, Carlos Eduardo García Sánchez ${ }^{1}$}

${ }^{1}$ Grupo de Investigación en Fluidos y Energía, Corporación Centro de Desarrollo Tecnológico del Gas, Bucaramanga, Colombia

\begin{abstract}
Static expansion systems are used to generate pressures in medium and high vacuum and are used in the calibration of absolute pressure meters in these pressure ranges. In the present study, the suitability of different models to represent the final pressures in a static expansion system with two tanks is analysed. It is concluded that the use of the ideal gas model is adequate in most simulated conditions, while the assumption that the residual pressure is zero before expansion presents problems under certain conditions. An uncertainty analysis of the process is carried out, which leads to evidence of the high importance of uncertainty in a first expansion over subsequent expansion processes. Finally, an analysis of the expansion system based on uncertainty is carried out to estimate the effect of the metrological characteristics of the measurements of the input quantities. Said design process can make it possible to determine a set of restrictions on the uncertainties of the input quantities.
\end{abstract}

\section{Section: RESEARCH PAPER}

Keywords: Modelling; pressure; static expansion; uncertainty

Citation: Carlos Mauricio Villamizar Mora, Jonathan Javier Duarte Franco, Victor Jose Manrique Moreno, Carlos Eduardo García Sánchez, Analysis of the mathematical modelling of a static expansion system, Acta IMEKO, vol. 10, no. 3, article 25, September 2021, identifier: IMEKO-ACTA-10 (2021)-03-25

Section Editor: Francesco Lamonaca, University of Calabria, Italy

Received January 29, 2021; In final form July 9, 2021; Published September 2021

Copyright: This is an open-access article distributed under the terms of the Creative Commons Attribution 3.0 License, which permits unrestricted use, distribution, and reproduction in any medium, provided the original author and source are credited.

Funding: This work was funded by Colombia's Servicio Nacional de Aprendizaje (SENA) through the Special Cooperation Agreement no. 0233 of 2018.

Corresponding author: Carlos Eduardo García Sánchez, e-mail: cgarcia@cdtdegas.com

\section{INTRODUCTION}

Pressure measuring instruments, like any other measuring device, require periodic calibrations, to monitor changes in their performance, and guarantee their comparability with other meters [1]. In simple terms, a calibration consists of establishing a relationship between the values given by measurement standards, and those given by an instrument under test [2]. In the case of vacuum pressure gauges, that is, that measure absolute pressure values lower than atmospheric pressure, a system that can produce specific vacuum pressure values is required, given the importance of comparing the measurements given by the standard and by the meter under test at different values of the measured variable [3]. In the calibration process, it is of utmost importance that the specific pressure values that are generated have a low uncertainty. Uncertainty is a characteristic of any measurement, indicating the level of doubt about the reported value [4]. In this way, a better comparability of meters that have been calibrated with the process in question can be guaranteed.
Currently, in Colombia there is a lack of absolute pressure calibration services in the medium and high vacuum regions. For this reason, the Centro de Desarrollo Tecnológico del Gas (CDT de Gas) has developed a static expansion system, which allows the generation of pressures in the medium and high vacuum ranges, making it possible to calibrate pressure gauges in those regions. This type of system has been implemented in multiple laboratories worldwide. The present study shows the mathematical design process of the system, through an evaluation of the possible models to represent the behavior of the gas inside the system, and the use of uncertainty to define restrictions on the input quantities of the system.

\section{STATE OF THE ART}

\subsection{Static expansion systems}

The pressure region between absolute zero (total absence of molecules) and atmospheric pressure is called "vacuum". In turn, vacuum is classified as coarse (from $3000 \mathrm{~Pa}$ to atmospheric pressure), medium (between $0.1 \mathrm{~Pa}$ and $3000 \mathrm{~Pa}$ ), high (from 
$1 \cdot 10^{-7} \mathrm{~Pa}$ to $0.1 \mathrm{~Pa}$ ) and ultra-high (less than $1 \cdot 10^{-7} \mathrm{~Pa}$ ) [5]. In general, there is no pressure measurement technology that covers all regions of interest [6]. Among the most accurate equipment for generating pressures in the medium and high vacuum ranges are static expansion systems, with which pressures as low as $10^{-6} \mathrm{~Pa}$ [7]-[9] can be obtained. Several metrology laboratories and national metrology institutes have developed static expansion systems [10]-[13]. The generation of vacuum pressures using static expansion of a gas is a mature technology [14], and much of the development in recent years in the topic has been related to a careful evaluation of possible causes of error and the estimation of the uncertainty of the final pressure [8], [10], [15]. Calibration and measurement capabilities with expanded uncertainties lower than $0.3 \%(k=2)$ using static expansion systems have been reported [15]. Nitrogen is commonly used as a gas for this type of calibration, although other inert gases could also be used [3], [7], [16].

Static expansion systems are a set of tanks of different dimensions, connected by pipes and valves. To generate low pressures with high precision, a volume of gas, with a defined pressure, is allowed to expand to a larger volume, previously at a pressure as close to zero as possible [17]. Figure 1 presents a simplified diagram of the static expansion process, using a small tank (where the initial pressure is set) and a large tank (to which the equipment to be calibrated is connected). In Figure 1 and equations included in this work, $V_{P}$ is the volume of the small tank, $V_{G}$ is the volume of the large tank, $T_{i}$ is the initial temperature of the process, $T_{f}$ is the final temperature of the process, $P_{i}$ is the initial pressure of the process in the small tank, $P_{i, G}$ is the initial pressure of the process in the large tank, which should be as close as possible to zero, and $P_{f}$ is the final pressure of the process. To further reduce the pressure, it is possible to repeat the expansion process, using the initial pressure resulting from the previous expansions [8]; the lower achievable limit is imposed by the level of vacuum that can be generated in the large tank, and by the effects of sorption and degassing in the tanks and the instruments under test [18].

Static expansion systems can be used as primary calibration standards, calculating the final pressure from the initial pressure and the volume ratios of the gas expansion processes performed. They can also be used to generate pressure but using a pressure gauge as a reference for calibration, in this case being a calibration by direct comparison [3], [16], [19]-[22]. It is important to note that several of the high vacuum pressure measurement technologies, such as Pirani gauges, exhibit high non-linearity with respect to pressure [3], which usually implies requiring several calibration points in each order of magnitude of pressure. Table 1 presents the fundamental characteristics of some static expansion systems developed by various national metrology institutes.

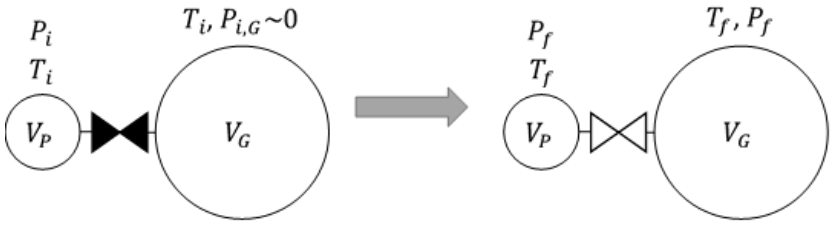

Figure 1. Static expansion process with two tanks. The initial state of the expansion process is shown on the left, and the final state on the right. It is assumed that there is spatial homogeneity of temperature in both tanks, but not necessarily temporal homogeneity.

Regarding the modelling of the process, some institutions have chosen to use the ideal gas model [13], [24], [25], while others have proposed the use of the virial equation as a real gas model for the expansion process [5], [7], [8], [23]. One aspect that is quite generalised is the assumption that the initial pressure in the calibration tank is zero, although the question remains whether this assumption is valid as the final pressure is smaller (that is, as the vacuum increases). Equation (1) presents the calculation of the pressure after a static expansion process, modelling the substance as an ideal gas and neglecting the initial pressure in the large tank [10], [13], [24], [25].

$$
P_{f}=P_{i} \frac{V_{P}}{V_{P}+V_{G}} \frac{T_{f}}{T_{i}} .
$$

On the other hand, (2) shows the calculation of the final pressure obtained with a static expansion, based on the truncated virial expansion in the second term and neglecting the initial pressure in the large tank [5], [7], [ 8], [23].

$$
P_{f}=P_{i} \frac{V_{P}}{V_{P}+V_{G}} \frac{T_{f}}{T_{i}} \frac{1+B_{f} \frac{P_{f}}{R T_{f}}}{1+B_{i} \frac{P_{i}}{R T_{i}}} .
$$

In (2) and following equations, $R$ is the molar constant of the gases, equal to $8.314462618 \mathrm{~J} \mathrm{~mol}^{-1} \mathrm{~K}^{-1}, B_{f}$ is the second virial coefficient of nitrogen in the conditions of the end of the process and $B_{i}$ is the second virial coefficient of nitrogen in the conditions of the beginning of the process. The second virial coefficient is a function of the substance or mixture of substances, and a function of temperature.

Another important aspect related to the initial pressure in the calibration tank is that to reach the residual pressure, or minimum pressure achievable in the calibration chamber, it is usually required to pump for many hours and to bake the tank [16]. Residual pressure is limited by pumping speed, by leaks, by gas desorption from materials exposed to vacuum, and by cleanliness of test gauges [1]. Baking refers to the heating of the chamber, to about $200{ }^{\circ} \mathrm{C}$, to desorb the gas from the internal

Table 1. Examples of static expansion systems developed in different national metrology institutes. It is not an exhaustive list: the PTB has another static expansion system in addition to the one mentioned here, and systems such as those of KRISS (from South Korea) or NPL (from England) were not included either.

\begin{tabular}{lccc}
\hline Institution & Approximate volumes of tanks (L) & Pressure range (Pa) & Reference \\
\hline L'Istituto Nazionale di Ricerca Metrologica (INRIM) & $0.01,0.5$ and 68 & $0.1-1000$ \\
Centro Nacional de Metrología (CENAM) & $0.5,1,50$ and 100 & $0.00001-1000$ \\
Centro Español de Metrología (CEM) & $0.5,1,1,100$ and 100 & $0.0001-1000$ \\
Physikalisch-Technische Bundesanstalt (PTB) & {$[23]$} & $0.000001-1000$ \\
TÜBITAK-Ulusal Metroloji Enstitüsü (UME) & $0.15,0.15,0.7,15,15$ and 72 & $0.0009-1000$ \\
\hline
\end{tabular}


walls of the materials, a necessary procedure to maintain ultrahigh and superior voids [18], [19]. Other aspects that have been studied in static expansion systems are the determination of tank volumes by methods other than gravimetry [13], [24] and the effect of the inhomogeneity of temperature in the tanks on the process [8].

\subsection{Uncertainty}

Uncertainty is the name given to the level of doubt that a measurement result has [2], [4], [12]. The two most common ways to report uncertainty are standard uncertainty, which represents the standard deviation of the probability distribution with which the measurement result is modeled, and expanded uncertainty, which is half the length of a coverage interval on the measurement result, with a specified coverage percentage (for example, $95 \%$ ). The most widely used method to estimate the uncertainty of a measurement result is the GUM method [4]. The uncertainty estimation process requires the clear establishment of the measurement model, which presents the way in which the measurand (quantity to be measured) is calculated from its input quantities. Subsequently, in the GUM method, the standard uncertainty $u(y)$ of the measurand $y$ will be estimated from the uncertainties $u\left(x_{1}\right), u\left(x_{2}\right), \ldots, u\left(x_{\mathrm{n}}\right)$ of the input variables $X_{1}$, $X_{2}, \ldots, X_{\mathrm{n}}$ using the measurement model $y=f\left(X_{1}, X_{2}, \ldots, X_{n}\right)$. Neglecting the correlation between the input quantities and the higher order terms, it is obtained the simplest version of the GUM method, in which the estimation of the standard uncertainty of the measurand is made according to (3) [4]:

$$
=\sqrt{\left(\frac{\partial f}{\partial x_{1}}\right)^{2} u^{2}\left(x_{1}\right)+\left(\frac{\partial f}{\partial x_{2}}\right)^{2} u^{2}\left(x_{2}\right)+\cdots+\left(\frac{\partial f}{\partial x_{n}}\right)^{2} u^{2}\left(x_{n}\right) .} .
$$

\section{METHODOLOGY}

In a two-tank static expansion process, the final pressure depends on the initial pressure in the small tank, the initial pressure in the large tank, the volumes of the tanks, the initial and final temperatures, and the nature of the gas that is expanding. The model used, however, may differ according to the assumptions made about the process, which can lead to the neglect of the effect of some variables. As mentioned in the state of the art, different institutions have opted for different models to calculate the pressures after the expansion processes. In the present work, three simplified models were compared against the complete model to calculate the resulting pressure after an expansion process, to define which of the models can be considered adequate to calculate the pressure after one or more expansion processes.

The most complete model to represent the process is based on a real gas model and considers both the initial pressure in the large tank and the inhomogeneity of temperatures at the beginning and at the end. It is well known that the ideal gas model represents the behavior of a gas when $P \rightarrow 0$, since with a non-existent pressure the assumptions of said model would be exactly fulfilled (the volumes of the molecules are negligible with respect to the total volume of the gas, the forces intermolecular tends to zero and molecular shocks are perfectly elastic) [26]. The ideal gas model is a convenient limiting case, which can be deduced from theoretical considerations, but it does not accurately represent the behavior in the gas phase of pure substances or mixtures that are at pressures other than zero [27].
Among the real gas models that have been developed, the virial equation of state has the desirable characteristic that its parameters can be related to intermolecular forces [27]. Considering the above, in the present work a model based on the virial equation to represent the behavior of the substance that undergoes expansion was established as the reference model for the final pressure after expansion. It was assumed spatial homogeneity (although not temporal) of temperature, considering that the static expansion system will eventually operate under controlled environmental conditions.

Two simplifications were evaluated: (1) using an ideal gas model instead of a real gas model, and (2) neglecting the initial pressure in the calibration tank. The main reason that would support the use of ideal gas is that this model is based on assumptions about the behavior of gaseous substances that are approximately satisfied at very low pressures [26]. Additionally, it is common to use nitrogen as a gas inside the expansion system, and the virial coefficient for this gas is very small; this coefficient may be relevant for other heavier inert gases [14]. Regarding the initial pressure in the large tank, in all the references consulted [5], [7], [8], [10], [13], [23], [24], [25] is neglected, but it is possible to wonder how much of an impact this assumption can have. In this way, four models were compared. Model 1 was the model without simplifications and was therefore taken as the reference model; this model is presented at the beginning of section 4 (Results and discussion). Model 2 was based on the ideal gas model and considered the initial pressure in the large tank. Model 3 was based on the real gas model but neglecting the initial pressure in the large tank. And Model 4 contained the two simplifying assumptions, that is, it was based on ideal gas and used zero as the initial pressure value in the calibration tank.

To make the comparisons, the final pressure with each of the four models was calculated, and the error in the pressure value of each of the last three models with respect to the reference one (Model 1) was determined. The error of models 2, 3 and 4 was calculated with (4), where $E_{i}$ is the percentage error made by the i-th model, $i=2,3,4, P_{f, 1}$ is the final pressure calculated with model 1 , and $P_{f, i}$ is the final pressure calculated with the $i$-th model:

$$
E_{i}=\left(\frac{P_{f, i}-P_{f, 1}}{P_{f, 1}}\right) \cdot 100 \%
$$

In order to consider a wide range of conditions, comparisons were made with the possible combinations of two volume relationships, two differences between initial and final temperatures, two initial pressures in the small tank, two initial pressures in the large tank, and four values of consecutive expansions.

After determining whether any of the simplified models was appropriate for the two-tank static expansion system, the GUM method was applied, without correlation or higher order terms, to estimate the uncertainty using the chosen model as the measurement model. The uncertainty budget, that is, the contribution of the different input quantities to the final pressure, was evaluated for different uncertainty values of said input quantities [4]. In this way, the importance of the different input magnitudes on the final pressure was evaluated, in a wide range of conditions. 


\section{RESULTS AND DISCUSSION}

By modelling the behaviour of nitrogen using the virial expansion truncated in the second term and considering the initial pressure in the large tank, the final pressure after a static expansion process is calculated by

$$
P_{f}=\frac{1+B_{f} \frac{P_{f}}{R T_{f}}}{\left(V_{P}+V_{G}\right)}\left[\frac{P_{i} V_{P}}{1+B_{i} \frac{P_{i}}{R T_{i}}}+\frac{P_{i, G} V_{G}}{1+B_{i} \frac{P_{i, G}}{R T_{i}}}\right] \frac{T_{f}}{T_{i}} .
$$

This model was called "Model 1" and is the reference model.

The main drawback of the model in (5) is that $P_{f}$ is an implicit variable, so it is required to solve the equation using a numerical method. In the present work, the secant method [28] was used to solve (5). It became evident that using as the starting point for the method the value of $P_{f}$ calculated with Model 4 (which is the simplest), the method required very few steps to achieve convergence.

Equation (6) presents the "Model 2", which consists of the resulting model for the final pressure after a static expansion process, using ideal gas to represent the behaviour of the substance and considering the initial pressure in the large tank:

$$
P_{f}=\frac{P_{i} V_{P}+P_{i, G} V_{G}}{V_{P}+V_{G}} \frac{T_{f}}{T_{i}} .
$$

The "Model 3" is (2) (shown in the "State of the art" section), which represents the calculation of the final pressure obtained with a static expansion, based on the virial expansion truncated in the second term and neglecting the initial pressure in the large tank. This model is implicit for $P_{f}$, just like Model 1 .

The "Model 4" is equation (1) (shown in the "State of the art"), which represents the calculation of the resulting pressure after a static expansion process modelling the substance as an ideal gas and neglecting the initial pressure in the large tank.

Sixty-four conditions were simulated, corresponding to the possible combinations of the following values of the input variables: two volume relationships (1:20 and 1:150), two differences between final and initial temperatures $(0 \mathrm{~K}$ and $5 \mathrm{~K})$, two initial pressures in the small tank $(10000 \mathrm{~Pa}$ and $50000 \mathrm{~Pa})$, two initial pressures in the large tank $(0.001 \mathrm{~Pa}$ and $0.00001 \mathrm{~Pa})$ and four consecutive expansion amounts (1,2,3 and 4).

The percentage errors in the calculation of the final pressure committed by the three models evaluated in each of the sixtyfour conditions are summarised in Figure 2. The highest error made with model 2 is $-0.0134 \%$, and it occurs under certain conditions by performing the expansion process only once. This behaviour is explained taking into account that the ideal gas model works better the lower the pressure, and the highest pressure values (the lowest vacuum levels) are obtained when performing a single expansion. In any case, the error is quite low, and depending on the target uncertainty in the final pressure, it is possible that model 2 can be used without problem. On the other hand, with models 3 and 4 very high errors are made under certain conditions, exceeding $-20 \%$ after three consecutive expansions, and reaching $-97 \%$ in some cases with four consecutive expansions. These very high errors occur when the initial pressure in the large tank is $1 \cdot 10^{-3} \mathrm{~Pa}$, which is an exaggeratedly high value considering the capabilities of current vacuum pumps, such as turbomolecular pumps, but that could occur if the system is not properly baked. In any case, considering the purpose of the analysis to evaluate the performance of the models under different conditions, the combinations of values of input quantities tested indicate that in some situations models 3 and 4 will have an unacceptable performance to determine the pressure of reference in a pressure gauge calibration process.
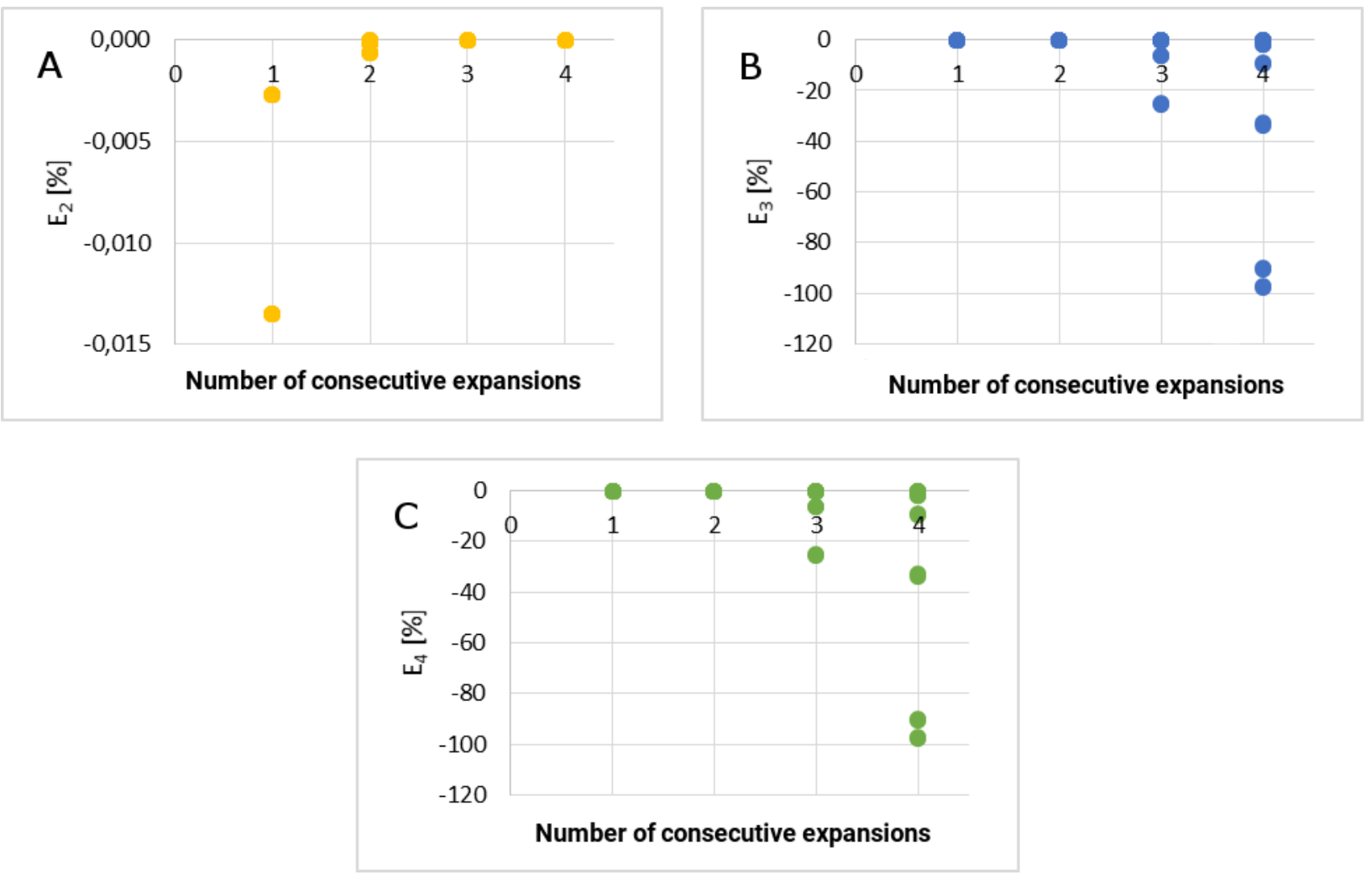

Figure 2. Percentage error in the final pressure calculated with the evaluated models, against the number of consecutive expansions. A: error made by model 2. B: error presented by model 3. C: resulting error when applying model 4. 
Table 2. Values of the input quantities and their uncertainties in the base study case defined for both uncertainty analysis. The standard uncertainty of each input quantity was $0.3 \%$ of the respective value of the quantity.

\begin{tabular}{ccc}
\hline Input quantity & Value & Standard uncertainty \\
\hline$P_{i}(\mathrm{~Pa})$ & 50000 & 45 \\
$P_{i, G}(\mathrm{~Pa})$ & 0.000010 & 0.000002 \\
$V_{P}\left(\mathrm{~m}^{3}\right)$ & 0.001000 & 0.000005 \\
$V_{G}\left(\mathrm{~m}^{3}\right)$ & 0.10000 & 0.00005 \\
$T_{i}(\mathrm{~K})$ & 296.15 & 0.30 \\
$T_{f}(\mathrm{~K})$ & 297.15 & 0.30 \\
\hline
\end{tabular}

Based on the results of the previous section, it was decided to use model 2 to perform the uncertainty analysis. The GUM equation applied to said model is shown in (7).

$$
\begin{aligned}
u\left(P_{f}\right)= & {\left[\left(\frac{\partial P_{f}}{\partial P_{i}}\right)^{2} u^{2}\left(P_{i}\right)+\left(\frac{\partial P_{f}}{\partial P_{i, G}}\right)^{2} u^{2}\left(P_{i, G}\right)\right.} \\
& +\left(\frac{\partial P_{f}}{\partial V_{P}}\right)^{2} u^{2}\left(V_{P}\right)+\left(\frac{\partial P_{f}}{\partial V_{G}}\right)^{2} u^{2}\left(V_{G}\right) \\
& \left.+\left(\frac{\partial P_{f}}{\partial T_{f}}\right)^{2} u^{2}\left(T_{f}\right)+\left(\frac{\partial P_{f}}{\partial T_{i}}\right)^{2} u^{2}\left(T_{i}\right)\right]^{0.5}
\end{aligned}
$$

To evaluate the generalities of the effect of the uncertainty of the input quantities on the uncertainty of the final pressure, a base case and six derived cases were considered. The base case is presented in Table 2. Four consecutive expansions were considered in the base case, so that the pressures after the first, second, third, and fourth consecutive expansion were 496.7 Pa, $4.935 \mathrm{~Pa}, 0.04903 \mathrm{~Pa}$, and $0.0004970 \mathrm{~Pa}$, respectively. In the six derived cases, the values of the input quantities remained identical to those of the base case, as were all the uncertainties except one, which was set at $1 \%$ of the value of the quantity. Table 3 presents the standard uncertainties, in terms of percentage of the value of the measurand, obtained after the different numbers of expansions tested in each of the 7 study cases. In all cases, the relative standard uncertainty increases as more expansions are made and the final pressure decreases. In the hypothetical base case, the uncertainty of the pressure values
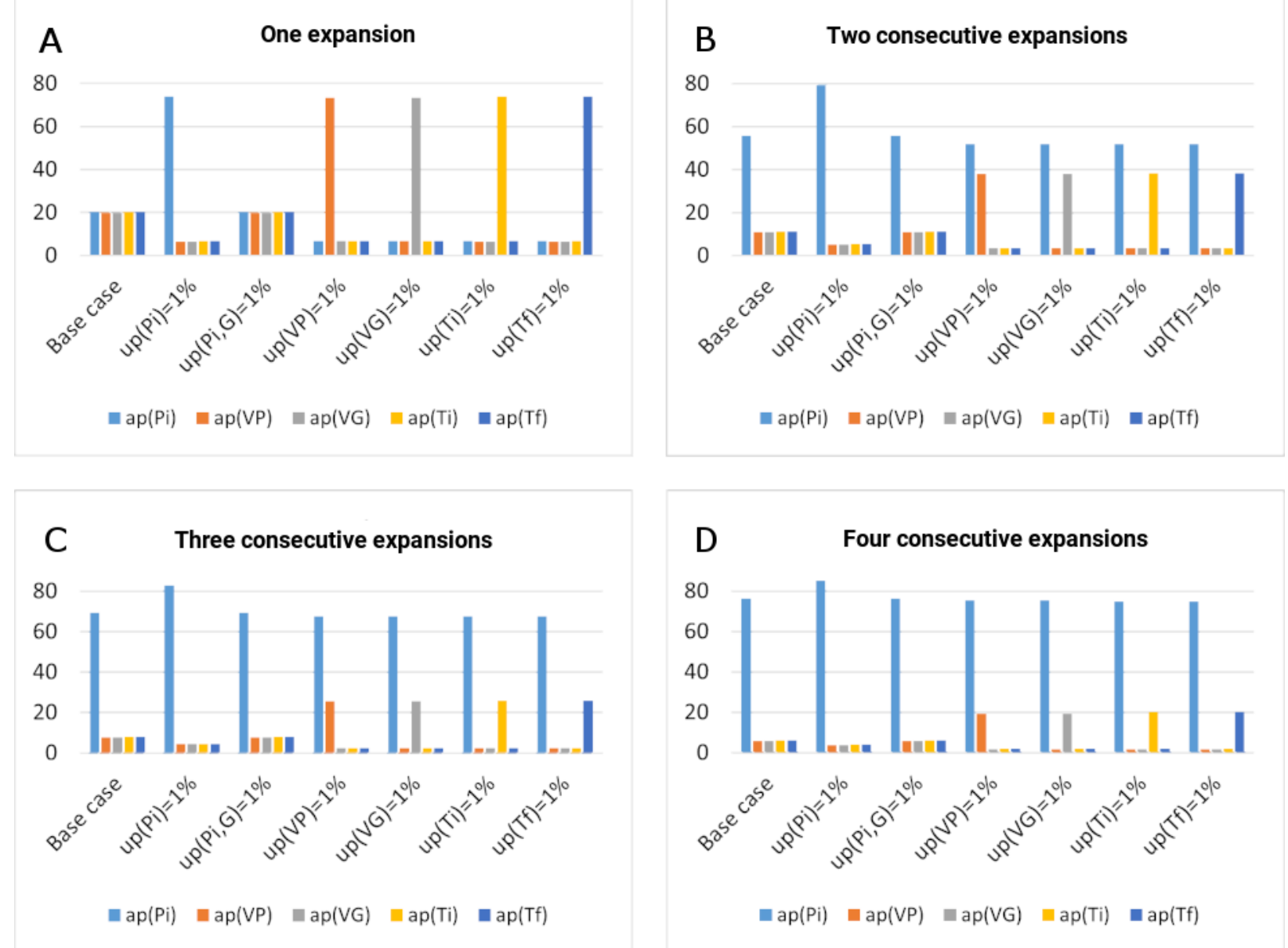

\section{Four consecutive expansions}

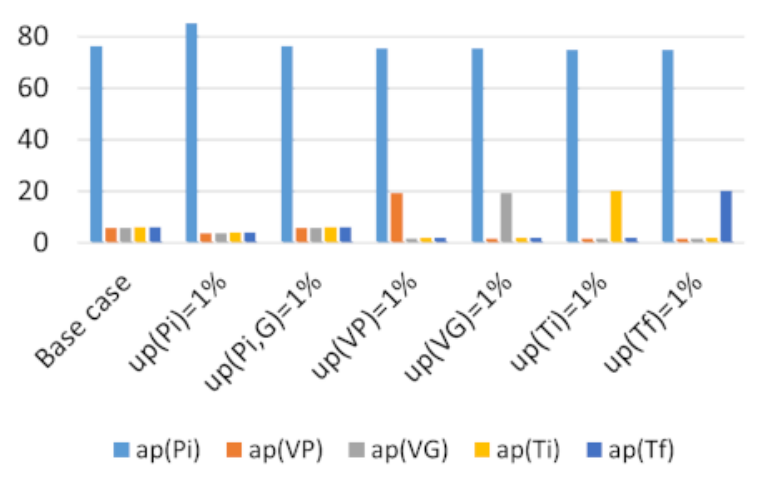

Figure 3. Percentage contributions of the uncertainties of the input quantities over the uncertainty of the final pressure ("uncertainty budget"), for the seven case studies, with four different numbers of consecutive expansions. The percentage contribution of the initial pressure in the large tank was omitted from the budgets, since it was less than $0.03 \%$ in all cases. A: one expansion. B: two expansions. C: three expansions. D: four expansions. 
Table 3. Relative standard uncertainty (\%) of the final pressure after several consecutive expansions, for the seven case studies proposed to review the impact of the input quantities.

\begin{tabular}{lcccc}
\hline Study case & First expansion & Second expansion & Third expansion & Fourth expansion \\
\hline Base case & 0.67 & 0.90 & 1.1 & 1.2 \\
$u\left(P_{i}\right)=0.01 \cdot P_{i}$ & 1.2 & 1.3 & 1.4 & 1.5 \\
$u\left(P_{i, G}\right)=0.01 \cdot P_{i, G}$ & 0.67 & 0.90 & 1.1 & 1.2 \\
$u\left(V_{P}\right)=0.01 \cdot V_{P}$ & 1.2 & 1.6 & 2.0 & 2.2 \\
$u\left(V_{G}\right)=0.01 \cdot V_{G}$ & 1.2 & 1.6 & 2.0 & 2.2 \\
$u\left(T_{i}\right)=0.01 \cdot T_{i}$ & 1.2 & 1.6 & 2.0 & 2.2 \\
$u\left(T_{f}\right)=0.01 \cdot T_{f}$ & 1.2 & 1.6 & 2.0 & 2.2 \\
\hline
\end{tabular}

obtained is very high (reaching $1.21 \%$ ), considering the possibilities of the system. It can also be seen that increasing the uncertainty from $0.3 \%$ to $1 \%$ for $V_{P}, V_{G}, T_{i}$ and $T_{f}$ has a practically identical effect on the pressure uncertainty, while the impact of this increase in uncertainty for $P_{i}$ is smaller as the number of consecutive expansions grows. On the other hand, increasing the uncertainty of $P_{i, G}$ has a negligible effect, for the values used in the base case.

Figure 3 presents the uncertainty budgets of the seven case studies. After a single expansion, the budgets of the base case and the case with $1 \%$ uncertainty for $P_{i, G}$ show a balanced contribution of the different input magnitudes, while for the other 5 cases the budget is dominated by the input quantity to which the uncertainty was increased. On the other hand, as the number of successive expansions increases, for all the study cases the role of the initial pressure of the expansion process (which is the final pressure reached in the previous expansion) gradually increases over the uncertainty of the resulting pressure. This fact indicates the high importance of uncertainty during the first expansion process over uncertainty in subsequent expansions.

Additionally, an uncertainty analysis was carried out for a case more adjusted to reality, taking values of the input magnitudes within the expected intervals, and assigning them uncertainties similar to those that can be obtained when using medium-high quality measurement instruments. Table 2 summarises the values used for that case. Four consecutive expansions were simulated, and the value of the final pressure, its uncertainty and the respective uncertainty budget were determined. The result is presented in Table 4.

This study case shows that the resulting pressure uncertainty grows from $0.72 \%$ with one expansion to $1.5 \%$ after the fourth expansion. It is also evidenced that the uncertainty of the final pressure is being dominated by the uncertainties of the volumes of the tanks, with the uncertainty of the volume of each of the tanks contributing $47.2 \%$ to the uncertainty of the pressure after expansion (and considering that the pressure after 2, 3 and 4 expansions is dominated by the initial pressure, that is, the final pressure of the previous expansion process). It is interesting that the contribution of the initial pressure in the large tank is only appreciable in the fourth expansion.

\section{CONCLUSIONS}

It was possible to evaluate the adequacy of the different models proposed. It was determined that the use of an ideal gas model instead of a real gas model caused a maximum error of $0.0135 \%$ on the pressure value, under the evaluated conditions (64 different conditions, between 1 and 4 expansion processes). In this way, depending on the uncertainty objective in the calibration process with the expansion system, it is possible that this simplification can be used without problems. On the other hand, neglecting the initial pressure in the calibration chamber can lead to errors in the pressure value of several tens in percentage, of even $97 \%$ under the evaluated conditions, especially as the number of consecutive expansions that take place increases. Therefore, it is concluded that it is preferable not to neglect the initial residual pressure in the calibration chamber, unless it is guaranteed that said pressure is maintained at $1 \cdot 10^{-7} \mathrm{~Pa}$ or less, with the baking processes and long periods of pumping that that requires.

Additionally, it was possible to analyse the effect of the uncertainty of the input quantities on the uncertainty of the final pressure after one or more consecutive expansions. It became evident that the magnitudes with the greatest influence on the final pressure obtained are the volumes of the tanks used in the expansion processes.

\section{ACKNOWLEDGEMENTS}

This work was financed by Colombia's Servicio Nacional de Aprendizaje (SENA) through the Special Cooperation Agreement no. 0233 of 2018. SENA's Centro Industrial del Diseño y la Manufactura and Centro Industrial y del Desarrollo Tecnológico participated in the project transfer plan.

Table 4. Results of the second uncertainty analysis.

\begin{tabular}{|c|c|c|c|c|c|c|c|c|}
\hline \multirow{2}{*}{$\begin{array}{l}\text { Expansion } \\
\text { number }\end{array}$} & \multirow{2}{*}{$\begin{array}{c}P_{f} \\
(\mathrm{~Pa})\end{array}$} & \multirow{2}{*}{$\begin{array}{c}\boldsymbol{u}\left(\boldsymbol{P}_{\boldsymbol{f}}\right) \\
(\mathrm{Pa})\end{array}$} & \multicolumn{6}{|c|}{ Contribution to the uncertainty budget in \% } \\
\hline & & & $\boldsymbol{P}_{\boldsymbol{i}}$ & $P_{i, G}$ & $V_{P}$ & $V_{G}$ & $T_{i}$ & $T_{f}$ \\
\hline 1 & 496.7 & 3.6 & 1.6 & 0.0 & 47.2 & 47.2 & 2.0 & 2.0 \\
\hline 2 & 4.935 & 0.050 & 50.4 & 0.0 & 23.8 & 23.8 & 1.0 & 1.0 \\
\hline 3 & 0.04903 & 0.00061 & 66.8 & 0.0 & 15.9 & 15.9 & 0.7 & 0.7 \\
\hline 4 & 0.0004970 & 0.0000073 & 69.4 & 7.5 & 11.1 & 11.1 & 0.5 & 0.5 \\
\hline
\end{tabular}




\section{REFERENCES}

[1] National Physical Laboratory, Guide to the measurement of pressure and vacuum, London, 1988.

[2] Joint Committee for Guides in Metrology, JCGM 200:2012 International vocabulary of metrology - Basic and general concepts and associated terms (VIM) - 3rd ed., Joint Committee for Guides in Metrology, 2012.

[3] R. E. Ellefson, A. P. Miller, Recommended practice for calibrating vacuum gauges of the thermal conductivity type, Journal of Vacuum Science \& Technology A 18(5) (2000), pp. 2568-2577. DOI: $10.1116 / 1.1286024$

[4] Joint Committee for Guides in Metrology, JCGM 100:2008 Evaluation of measurement data - Guide to the expression of uncertainty in measurement, Joint Committee for Guides in Metrology, 2008.

[5] S. Ruiz González, Desarrollo de un nuevo patrón nacional de presión - desde la columna de mercurio a patrones primarios de vacío (tesis doctoral), Universidad de Valladolid, Valladolid, España, 2000.

[6] B. G. Lipták, Instrument Engineers' Handbook - Fourth Edition - Process Measurement and Analysis - Volume I, CRC Press LLC, Boca Raton, 2003.

[7] J. C. Greenwood, Simulation of the operation and characteristics of static expansion pressure standards, Vacuum 80 (2006), pp. 548-553.

DOI: $10.1016 /$ i.vacuum.2005.09.003

[8] W. Jitschin, High-accuracy calibration in the vacuum range 0.3 Pa to $4000 \mathrm{~Pa}$ using the primary standard of static gas expansion, Metrologia 39 (2002), pp. 249-261. DOI: $10.1088 / 0026-1394 / 39 / 3 / 2$

[9] N. Medina, S. Ruiz González, C. Matilla, Developments in the pressure field at CEM, IMEKO 20th TC3, 3rd TC16 and 1st TC222 International Conference - Cultivating metrological knowledge, Mérida, Mexico, 2007. Online [Accessed 8 September 2021]

https://www.imeko.org/publications/tc16-2007/IMEKOTC16-2007-036u.pdf

[10] M. Astrua, D. Mari, S. Pasqualin, Improvement of INRiM static expansion system as vacuum primary standard between $10^{-4} \mathrm{~Pa}$ and $1000 \mathrm{~Pa}$, 19th International Congress of Metrology, 2019, pp. 27007.

DOI: $10.1051 /$ metrology $/ 20192700$

[11] J. C. Torres Guzmán, L. A. Santander Romero, K. Jousten, Realization of the medium and high vacuum primary standard in CENAM, Mexico, Metrologia 42 (2005), pp. S157-S160. DOI: $10.1088 / 0026-1394 / 42 / 6 /$ S01

[12] S. Phanakulwiiit, J. Pitakarnnop, Establishment fo Thailand's national primary vacuum standard by a static expansion method, Journal of Physics: Conference Series 1380 (2019), pp. 012003. DOI: $\underline{10.1088 / 1742-6596 / 1380 / 1 / 012003}$

[13] D. Herranz, A. Pérez, Realización de un sistema de expansión estática como patrón nacional de presión absoluta en el rango de $10^{-4}$ a $1000 \mathrm{~Pa}$, Jornada de Difusión de Resultados de Proyectos CEM, Madrid, España, 2010.

[14] K. F. Poulter, The calibration of vacuum gauges, Journal of Physics E: Scientific Instruments 10(2) (1977), pp. 112-125. DOI: $\underline{10.1088 / 0022-3735 / 10 / 2 / 002}$
[15] Y. Takei, H. Yoshida, E. Komatsu, K. Arai, Uncertainty evaluation of the static expansion system and its long-term stability at NMIJ, Vacuum 187110034.

DOI: $10.1016 /$ i.vacuum.2020.110034

[16] International Organization for Standardization, International Standard ISO 3567 - Vacuum gauges - Calibration by direct comparison with a reference gauge, International Organization for Standardization, 2011.

[17] D. Herranz, S. Ruiz González, N. Medina, Volume ratio determination in static expansion systems by means of two pressure balances, XIX IMEKO World Congress - Fundamental and Applied Metrology, Lisboa, Portugal, 2009. Online [Accessed 8 September 2021] https://www.imeko.org/publications/wc-2009/IMEKO-WC2009-TC16-280.pdf

[18] W. Steckelmacher, The calibration of vacuum gauges, Vacuum 37(8-9) (1987), pp. 651-657. DOI: $10.1016 / 0042-207 X(87) 90051-0$

[19] Centro Español de Metrología, Procedimiento ME-001 para la calibración de medidores de vacío - Edición digital 1, Centro Español de Metrología, Madrid, 2011.

[20] J. A. Fedchak, P. J. Abbott, J. H. Hendricks, P. C. Arnold, N. T. Peacock, Review Article: Recommended practice for calibrating vacuum gauges of the ionization type, Journal of Vacuum Science \& Technology A 36(3) (2018), pp. 030802. DOI: $\underline{10.1116 / 1.5025060}$

[21] International Organization for Standardization, International Standard ISO 19685 - Vacuum gauges - Vacuum gauges Specifications, calibration and measurement uncertainties for Pirani gauges, International Organization for Standardization, Switzerland, 2017.

[22] P. Semwal, Z. Khan, K. R. Dhanani, F. S. Pathan, S. George, D. C. Raval, P. L. Thankey, Y. Paravatsu, M. Himabindu, Spinning rotor gauge based vacuum gauge calibration system at the Institute for Plasma Research (IPR), Journal of Physics: Conference Series 390 (2012), pp. 012027. DOI: $\underline{10.1088 / 1742-6596 / 390 / 1 / 012027}$

[23] S. Cardona B., J. C. Torres Guzmán, L. Santander Romero, Sistema de referencia nacional para la medición de vacío, Simposio de Metrología 2001 CENAM, Querétaro, México, 2001.

[24] K. Jousten, P. Röhl, V. M. Aranda Contreras, Volume ratio determination in static expansion systems by means of a spinning rotor gauge, Vacuum 52 (1999), pp. 491-499. DOI: $10.1016 /$ S0042-207X(98)00337-6

[25] R. Kangi, B. Ongun, A. Elkatmis, The new UME primary standard for pressure generation in the range from $9 \times 10^{-4}$ to $10^{3} \mathrm{~Pa}$, Metrologia 41 (2004), pp. 251-256. DOI: $10.1088 / 0026-1394 / 41 / 4 / 005$

[26] J. M. Smith, H. C. Van Ness, M. M. Abbott, M. M. Introducción a la termodinámica en ingeniería química - 7th ed., McGraw-Hill Interamericana, Mexico, 2007.

[27] J. M. Prausnitz, R. N. Lichtenthaler, E. Gomes de Azevedo, Termodinámica molecular de los equilibrios de fase - 3rd ed., Prentice-Hall, Madrid, 2000.

[28] R. L. Burden, J. D. Faires. Numercal Analysis - 9th ed., Brooks/Cole, Cengage Learning, USA, 2011. 